\title{
A unified analytical solution of the steady-state atmospheric diffusion equation
}

\author{
J.S. Pérez Guerrero ${ }^{\text {a,* }}$, L.C.G. Pimentel ${ }^{\text {b,c }}$, J.F. Oliveira-Júnior ${ }^{c}$, P.F.L. Heilbron Filho ${ }^{\mathrm{a}}$, A.G. Ulke ${ }^{\text {d,e }}$ \\ ${ }^{a}$ Brazilian Nuclear Energy Commission (CNEN), Rua General Severiano 90, CEP 22290-901, RJ-Rio de Janeiro, Brazil \\ ${ }^{\mathrm{b}}$ Department of Meteorology, IGEO, Federal University of Rio de Janeiro, CCMN, Bloco H, 21941-916, RJ-Rio de Janeiro, Brazil \\ ${ }^{\mathrm{c}}$ Department of Mechanical Engineering, COPPE, Federal University of Rio de Janeiro, Centro de Tecnologia, Bloco G, 21945-970, RJ-Rio de Janeiro, Brazil \\ d Departamento de Cs. de la Atmósfera y los Océanos, Facultad de Cs. Exactas y Naturales, Universidad de Buenos Aires, Pabellón II, Piso 2, 1428 Ciudad Universitaria, \\ Buenos Aires, Argentina \\ ${ }^{\mathrm{e}}$ Unidad Mixta Internacional (UMI) - Instituto Franco Argentino sobre Estudios de Clima y sus Impactos (IFAECI)/CNRS, Argentina
}

\section{A R T I C L E I N F O}

\section{Article history:}

Received 2 December 2011

Received in revised form

29 February 2012

Accepted 5 March 2012

\section{Keywords:}

Atmospheric diffusion equation

Analytical solution

Integral transform

Associated eigenvalue problem

\begin{abstract}
A B S T R A C T
A unified analytical solution of the steady-state atmospheric diffusion equation for a finite and semiinfinite/infinite media was developed using the classic integral transform technique (CITT) which is based on a systematized method of separation of variable.

The solution was obtained considering an arbitrary mean wind velocity depending on the vertical coordinate $(z)$ and a generalized separable functional form for the eddy diffusivities in terms of the longitudinal $(x)$ and vertical coordinates $(z)$.

The examples described in this article show that the well known closed-form analytical solutions, available in the literature, for both finite and semi-infinite/infinite media are special cases of the present unified analytical solution. As an example of the strength of the developed methodology, the Copenhagen and Prairie Grass experiments were simulated (finite media with the mean wind speed and the turbulent diffusion coefficient described by different functional forms). The results indicate that the present solutions are in good agreement with those obtained using other analytical procedures, previously published in the literature. It is important to note that the eigenvalue problem is associated directly to the atmospheric diffusion equation making possible the development of the unified analytical solution and also resulting in the improvement of the convergence behavior in the series of the eigenfunctionexpansion.
\end{abstract}

(c) 2012 Elsevier Ltd. All rights reserved.

\section{Introduction}

Several phenomena in the atmospheric environment involve dispersion processes and interactions among components of the climate system. Consequently, a key issue in environmental studies is the correct representation of these processes to gain knowledge on transference mechanisms and their implications on short or long terms. The dispersion processes mainly take place in the atmospheric boundary layer and involve different transport mechanisms of the following quantities: Momentum, energy, and mass.

Different methodologies have been applied by many scientists to better understand these complicated mechanisms and properly represent them. Among them, the use of the species conservation equation and its solution using different techniques combined with

\footnotetext{
* Corresponding author. Tel.: +5521 2173 2618; fax: +5521 21732673.

E-mail address: jperez@cnen.gov.br (J.S. Pérez Guerrero).
}

observational data has been proved useful. The well known atmospheric diffusion equation can be established considering the Eulerian approach associated with the $\mathrm{K}$ theory or gradient transport hypothesis, one of the most widely used methodologies (Pasquill and Smith, 1983). The widespread use of this theory is due to its simplicity and the good agreement with the experimental data found in literature. The solution of the atmospheric diffusion equation is strongly dependent on the functional forms adopted for the wind speeds and the eddy diffusivities and can be obtained using numerical methods (finite differences, finite elements, etc.), hybrid or semi-analytical methods (as spectral methods, generalized integral transform technique, etc.) or by analytical methods (Green's function methods, classical integral transform technique).

A variety of Eulerian Air Quality computational models such as Community Multi-scale Air quality Model - CMAQ (Byun and Ching, 1999), Weather Research and Forecasting Chemistry WRF/CHEM (Grell et al., 2005) and chemistry-transport model CHIMERE (Bessagnet et al., 2008) are being improved and applied worldwide. These models are capable of dealing with complex 
phenomena such as photochemical kinetic mechanisms and aerosol formation processes; they incorporate the state-of-the-art in the chemical and physical processes involved in the multi-pollutant atmospheric diffusion. However, they do have high computational cost and input data needs for the correct simulation of such complex phenomena.

The ongoing need of appropriate tools to promptly answer the actual demands for regulatory and screening application or accidental releases of pollutants show the importance of continuous improvement and extension of the developed models as was the case of the last generation of Gaussian plume models such as: AERMIC Model - AERMOD (EPA, 2005) and Atmospheric Dispersion Modeling System ADMS (Carruthers et al., 1999).

Therefore, there is a real necessity to develop quick methods for the solution of the atmospheric diffusion equation with the objective of evaluating chemical and physical mechanisms models in the atmosphere and turbulent eddy parameterizations. As discussed by Russell and Dennis (2000), there is a great necessity to develop physical parameterizations for Atmospheric Quality Model - AQM involving turbulence closure problems.

In addition, a simple model is suitable to carry on sensitivity analysis for the different physico-chemical mechanisms with a more effective control, facilitating the evaluation of the developed methodology as well as the analysis and development of improved functional forms for the different mechanisms involved. This cannot be easily done in the case of complex models where the nonlinear interaction between the different components and the combined errors can result in false conclusions about the results of the model.

Analytical solutions of advective-diffusive transport problems continue to be of interest in many areas of science and engineering (Naveira-Cotta et al., 2009; Pérez Guerrero et al., 2009a,b, 2010; Pérez Guerrero and Skaggs, 2010; Almeida et al., 2008; Sharan and Modani, 2005; Tirabassi et al., 2008; Moreira et al., 2009 and Cassol et al., 2009). They are useful for a variety of applications, such as: Providing initial or approximate analyses of alternative pollution scenarios, conducting sensitivity analyses to investigate the effects of various parameters or processes involved in contaminant transport, extrapolation over large times and distances where numerical solutions may be impractical, serving as screening models or benchmark solutions for more complex transport processes that cannot be solved exactly, and for validating more comprehensive numerical solutions of the governing transport equations.

A number of solutions have been reported in the literature for transient and steady-state regimes: Two and three-dimensional formulations with infinite, semi-infinite or finite domain. Typically, in these modeling studies the wind speed velocity and eddy diffusivities were considered constants and/or polynomials. The source terms (point, line and area) were modeled using the Dirac's delta generalized function. We can mention some relevant analytical solutions such as those obtained by: Roberts (1923), Sutton (1932, 1943, 1949), Bosanquet and Pearson (1936), Frost (1946), Davies (1947), Yih (1952), Rounds (1955), Smith (1957), Berlyand (1975), Lebedeff and Hameed (1975), Yeh (1975), Demuth (1978), Huang (1979), Llewelyn (1983), Sharan et al. (1996), Lin and Hildemann (1996), Sharan and Gupta (2002), Sharan and Modani (2005, 2006), Sharan and Kumar (2009), Park and Baik (2008). More analytical solutions and references on this topic can be found, for example, in Sutton (1953), Hanna et al. (1982), Pasquill and Smith (1983), Seinfeld and Pandis (1998) and Byun et al. (2003).

Lately, a new group of non-power law analytical solutions have been obtained for more complicated and realistic functional forms of the variation of the mean wind velocity and vertical eddy diffusion (Ma and Daggupaty, 1998; Byun et al., 2003; Moreira et al., 2005, 2009; Wortmann et al., 2005; Almeida et al., 2008 and Kumar and Sharan, 2010).
The main difficulty in obtaining analytical solutions of the atmospheric diffusion equation is because the mathematical procedures tend to be relatively complicated, requiring difficult or tedious derivations and manipulations. However, the development of symbolic manipulation software such as Mathematica (Wolfram Research, Inc., 2009) has made these solution procedures more tractable.

The development of new analytical solutions with such systematized procedures as the classic integral transform techniques - CITT (Ozisik, 1980; Mikhailov and Ozisik, 1984) provides an efficient and straightforward approach for the solution of both transient and steady-state problems, with homogeneous and nonhomogeneous boundary conditions. A large variety of heat and mass diffusion problems have been categorized and treated systematically using this technique, creating a unified approach for solving those problems (Mikhailov and Ozisik, 1984). Transport equations not immediately analytically solvable with the CITT can often be transformed into an amenable form using techniques such as algebraic substitution or integrating factor methods (e.g. Pérez Guerrero et al., 2009a,b, 2010 and Pérez Guerrero and Skaggs, 2010).

The solution from CITT can be obtained based on the following steps (Cotta, 1993):

a) Choose an appropriate auxiliary eigenvalue problem and find the associated eigenvalues, eigenfunctions, norm, and orthogonalization property;

b) Develop the integral and inverse transforms;

c) Transform the partial differential equation into a system of ordinary differential or algebraic equations;

d) Solve the ordinary differential or algebraic system;

e) Use the inverse transform to obtain the unknown function.

The present work developed a unified analytical solution of the atmosphere diffusion problem for both finite and semi-infinite/ infinite domains with high rate of convergence of the results (measured using the number of terms in the series solution), for diverse atmospheric stability classes. The solution admits parameterizations such as the $u(z)$ type for the mean wind velocity and the $b(x) K(z)$ type for turbulent diffusion. The results show that the Gaussian plume models and the non-Gaussian models available in literature (Vaughan, 1961; Berlyand, 1975; Yeh, 1975; Huang, 1979 and Lin and Hildemann, 1996) are sub-cases of the unified procedure developed.

\section{Problem formulation}

The steady-state concentration distribution $c \equiv c(x, y, z)$ of a chemically inert species, released from a continuous source of strength $Q$ located at a specified position, $\left(x_{s}, y_{s}, z_{s}\right)$, can be described by the Eulerian steady-state atmospheric diffusion equation:

$$
\begin{gathered}
u(x, y, z) \frac{\partial c}{\partial x}+v(x, y, z) \frac{\partial c}{\partial y}+w(x, y, z) \frac{\partial c}{\partial z}=Q \delta\left(x-x_{s}\right) \delta\left(y-y_{s}\right) \delta\left(z-z_{s}\right) \\
+\frac{\partial}{\partial x}\left[K_{x x}(x, y, z) \frac{\partial c}{\partial x}\right]+\frac{\partial}{\partial y}\left[K_{y y}(x, y, z) \frac{\partial c}{\partial y}\right]+\frac{\partial}{\partial z}\left[K_{z z}(x, y, z) \frac{\partial c}{\partial z}\right]
\end{gathered}
$$

Where: $u(x, y, z), v(x, y, z), w(x, y, z)$ and $K_{x x}(x, y, z), K_{y y}(x, y, z), K_{z z}(x$, $y, z)$ respectively are, the wind speeds and the eddy diffusion coefficients along $x, y$ and $z$ directions and $\delta$ 's the Dirac delta generalized function.

It is assumed that the $x$ direction is aligned with the wind flow that only depends on the height, the turbulent diffusion in the direction of the mean wind is neglected compared to the advective transport mechanism and the source is located at $x_{s}=0$, i.e., 
$u(x, y, z)=u(z)$

$v(x, y, z)=0$

$w(x, y, z)=0$

$u(x, y, z) \frac{\partial c}{\partial x}>>\frac{\partial}{\partial x}\left[K_{x x}(x, y, z) \frac{\partial c}{\partial y}\right]$

It is also considered that the functional forms of the eddy diffusivities obey the following separable structure:

$K_{y}(x, z)=K_{y y}(x, y, z)=a(x) u(z)$

$K_{z}(x, z)=K_{z z}(x, y, z)=b(x) K(z)$

The functional form adopted in Eq. (3a) allows a Gaussian distribution for the transversal concentration.

Making the substitution on Eq. (1) results:

$u(z) \frac{\partial c}{\partial x}=K_{y}(x, z) \frac{\partial^{2} c}{\partial y^{2}}+\frac{\partial}{\partial z}\left[K_{z}(x, z) \frac{\partial c}{\partial z}\right]+Q \delta(x) \delta\left(y-y_{s}\right) \delta\left(z-z_{s}\right)$

The required initial and boundary conditions to complete the problem for a finite domain are:

$u(z) c(0, y, z)=0$

$K_{y}(x, z) \frac{\partial c\left(x,-y_{1}, z\right)}{\partial y}=0$

$K_{y}(x, z) \frac{\partial c\left(x, y_{1}, z\right)}{\partial y}=0$

$K_{z}\left(x, z_{0}\right) \frac{\partial c\left(x, y, z_{0}\right)}{\partial z}=0$

$K_{z}\left(x, z_{1}\right) \frac{\partial c\left(x, y, z_{1}\right)}{\partial z}=0$

Where: $\left[-y_{1}, y_{1}\right]$ is the domain in the $y$ direction, $z_{0}$ the roughness length and $z_{1}$ the top of the atmospheric boundary layer.

Because the boundary conditions are homogeneous and the source term is described by a Dirac delta function, the transport Eq. (4) and the initial condition (Eq. (5)) can be re-written in the following equivalent form (Seinfeld and Pandis, 1998; Duffy, 2001):

$u(z) \frac{\partial c}{\partial x}=K_{y}(x, z) \frac{\partial^{2} c}{\partial y^{2}}+\frac{\partial}{\partial z}\left[K_{z}(x, z) \frac{\partial c}{\partial z}\right]=L c$

And the operator $L(.) \equiv K_{y}(x, z) \partial^{2}(.) / \partial y^{2}+\partial / \partial z\left[K_{z}(x, z) \partial(.) / \partial z\right]$

$u(z) c(0, y, z)=Q \delta\left(y-y_{s}\right) \delta\left(z-z_{s}\right)$

With the boundary conditions (Eqs. (6a,b) and (7a,b)) remaining the same.

\section{Analytical solution}

The solution of the Eqs. (6a,b)-(9) is obtained applying the Classic Integral Transform Technique (CITT) following the systematized procedure given by Ozisik (1980) and Mikhailov and Ozisik (1984) as follows.

\subsection{The auxiliary eigenvalue problem}

Many eigenvalue problems can be associated with Eq. (8) and subject to Eqs. (6a,b) and (7a,b) in an integral transform procedure. However, it is more adequate to use the directly associated eigenvalue problem of the original problem because it allows an exact integral transformation:

$L \psi+\lambda(x)^{2} u(z) \psi=0$

$\frac{\partial \psi\left(-y_{1}, z\right)}{\partial y}=0$

$\frac{\partial \psi\left(y_{1}, z\right)}{\partial y}=0$

$\frac{\partial \psi\left(y, z_{0}\right)}{\partial z}=0$

$\frac{\partial \psi\left(y, z_{1}\right)}{\partial z}=0$

Where: $\psi \equiv \psi(y, z)$ and $\lambda(x)$ are the eigenfunction and eigenvalue respectively. The parametric dependence of the eigenvalue with $x$ is because the operator $L$ has coefficients depending on $x$.

This eigenvalue problem can be solved by the method of separation of variables:

$\psi(y, z)=Y(y) Z(z)$

Thus, using Eq. (3a,b) and Eq. (13) in Eq. (10) and grouping conveniently results:

$a(x) \frac{d^{2} Y / d y^{2}}{Y}+b(x) \frac{1}{u(z) Z} \frac{d}{d z}\left[K(z) \frac{d Z}{d z}\right]+\lambda(x)^{2}=0$

The following eigenvalue problems are obtained:

$\frac{d^{2} Y}{d y^{2}}+\gamma^{2} Y=0$

$\frac{d Y\left(-y_{1}\right)}{d y}=0$

$\frac{d Y\left(y_{1}\right)}{d y}=0$

$\frac{d}{d z}\left[K(z) \frac{d Z}{d z}\right]+\eta^{2} u(z) Z=0$

$\frac{d Z\left(z_{0}\right)}{d z}=0$

$\frac{d Z\left(z_{1}\right)}{d z}=0$

Where: $\gamma$ and $\eta$ are the separation constants and the eigenvalue $\lambda(x)$ is given by:

$\lambda^{2}(x)=\gamma^{2} a(x)+\eta^{2} b(x)$

The solutions of Eqs. $(15 a-c)$ and $(16 a-c)$ give a set of linearly independent eigenfunctions for $Y_{i}(y)$ and $Z_{j}(z)$ and consequently for $\psi_{i}(y, z)$. It can be noted that for each value of $\gamma_{i}$ and $\eta_{j}$ there is an associated infinite set of real eigenvalues $\lambda_{i} \equiv \lambda_{i j}(i=0,1,2, \ldots, \infty)$ 
The orthogonality properties of the eigenfunctions $Y_{i}(y)$ and $Z_{i}(z)$ are:

$$
\begin{gathered}
\int_{-y_{1}}^{y_{1}} Y_{i}(y) Y_{j}(y) d y=N_{y}\left(\gamma_{i}\right) \delta_{i, j} \\
\int_{z_{0}}^{z_{1}} u(z) Z_{i}(z) Z_{j}(z) d z=N_{z}\left(\eta_{j}\right) \delta_{i, j}
\end{gathered}
$$

Where: $\delta_{i, j}$ is the Kronecker delta, $N_{y}\left(\gamma_{i}\right)$ and $N_{z}\left(\eta_{i}\right)$ are, respectively, the norms of $Y_{i}(y)$ and $Z_{i}(z)$.

The eigenfunctions $\psi_{i}$ of this eigenvalue problem satisfying the following orthogonality property (Mikhailov and Ozisik, 1984) in the region $R$ delimited by the boundaries:

$$
\begin{aligned}
\int_{R} u(z) \psi_{i}(y, z) \psi_{j}(y, z) d y d z & =\int_{-y_{1}}^{y 1} \int_{z_{0}}^{z_{1}} u(z) \psi_{i}(y, z) \psi_{j}(y, z) d y d z \\
& =\bar{N}_{i} \delta_{i, j}
\end{aligned}
$$

Where: $\bar{N}_{i}=N_{y}\left(\gamma_{i}\right) N_{z}\left(\eta_{j}\right)$ is the norm of $\psi_{i}(y, z)$.

The solution of the eigenvalue problem of Eq. $(15 a-c)$ can be easily obtained:

$$
\begin{aligned}
& Y_{i}(y)=\cos \left[\gamma_{i}\left(y+y_{1}\right)\right] \\
& \gamma_{i}=\frac{i \pi}{2 y_{1}}(i=0,1,2, . .) \\
& N_{y}\left(\gamma_{i}\right)=\left\{\begin{array}{cc}
2 y_{1} & (i=0) \\
y_{1} & (i=1,2,3, \ldots)
\end{array}\right.
\end{aligned}
$$

On the other hand, a closed-form analytical solution for the eigenvalue problem of Eq. $(16 a-c)$ is guaranteed only for special functional forms of $u(z)$ and $K(z)$, where the eigenvalue problem can be reduced to the generalized Bessel equation (Mikhailov and Ozisik, 1984; Duffy, 2001). For general functional forms of $u(z)$ and $K(z)$ the solution of the eigenvalue problem could be obtained using the semi-analytic sign-count method (Mikhailov and Vulchanov, 1983) or from algorithms for automatic computation of eigenvalue and eigenfunctions of Sturm-Liouville problems (Bailey et al., 1978; Marletta and Pryce, 1992).

Another alternative solution procedure, followed in the present work, is through the application of the GITT (Mikhailov and Cotta, 1994), where the ordinary differential equation of the eigenvalue problem is reduced to an algebraic system, which is solved accurately and automatically by existing codes.

\subsection{The integral transform pair}

The unknown function $c(x, y, z)$ is represented by an eigenfunction-expansion series in terms of the eigenfunctions $\psi_{i}(y, z)$. Then, using the orthogonality property (Eq. (20)), the following integral transform pair is developed:

$\bar{c}_{i}(x)=\int_{R} u(z) \psi_{i}(y, z) c(x, y, z) d y d z$ (Transform)

$c(x, y, z)=\sum_{i=0}^{\infty} \frac{\psi_{i}(y, z)}{\bar{N}_{i}} \bar{c}_{i}(x)$ (Inverse)
Where: $\bar{c}_{i}(x)$ is the transformed "potential".

\subsection{Integral transform of the differential equation}

Applying in Eq. (8) the inverse formula (Eq. (23)) and considering the eigenvalue problem (Eq. (10)) results:

$\frac{\partial}{\partial x} \sum_{j=0}^{\infty} u(z) \frac{\psi_{j}(y, z)}{\bar{N}_{j}} \bar{c}_{j}(x)=\sum_{j=0}^{\infty} \frac{-\lambda(x)^{2} u(z) \psi_{j}(y, z)}{\bar{N}_{j}} \bar{c}_{j}(x)$ Now, operating both sides of the last equation with $\int_{R} \psi_{j}(y, z) d y d z$
and grouping appropriately:

$$
\begin{aligned}
& \frac{\partial}{\partial x} \sum_{i=0}^{\infty} \frac{\bar{c}_{i}(x)}{\bar{N}_{i}} \int_{R} u(z) \psi_{i}(y, z) \psi_{j}(y, z) d y d z \\
& \quad=-\sum_{i=0}^{\infty} \frac{\bar{c}_{i}(x)}{\bar{N}_{i}} \lambda_{i}(x)^{2} \int_{R} u(z) \psi_{i}(y, z) \psi_{j}(y, z) d y d z
\end{aligned}
$$

Then, considering the orthogonality expression (Eq. (20)) in Eq. (25) the following ordinary differential system is obtained:

$\frac{d \bar{c}_{i}(x)}{d x}=-\lambda_{i}(x)^{2} \bar{c}_{i}(x)$

The initial condition (Eq. (9)) is also transformed to yield:

$\bar{c}_{i}(0)=\int_{R} u(z) \psi_{i} c(0, y, z) d y d z$

\subsection{Analytical solution for the transformed and original problems}

Eqs. (26) and (27) are a set of decoupled ordinary differential equations, whose analytical solution is:

$\bar{c}_{i}(x)=\bar{c}_{i}(0) \exp \left[-\int_{0}^{x} \lambda_{i}^{2}\left(x^{\prime}\right) d x^{\prime}\right]$

Introducing the transformed potential $\bar{c}_{i}(x)$ into the inversion formula (Eq. (23)), we obtain the formal solution of the atmospheric diffusion equation:

$c(x, y, z)=\sum_{i=0}^{\infty} \frac{\psi_{i}(y, z)}{\bar{N}_{i}} \bar{c}_{i}(0) \exp \left[-\int_{0}^{x} \lambda_{i}^{2}\left(x^{\prime}\right) d x^{\prime}\right]$

Now by setting (Ozisik, 1980): $\psi_{i}(y, \quad z) \rightarrow Y(y) Z(z)$, $\lambda_{i}^{2}(x) \rightarrow \gamma_{i}^{2} a(x)+\eta_{j}^{2} b(x), \quad \sum_{i=0}^{\infty} \rightarrow \sum_{i=0}^{\infty} \sum_{j=0}^{\infty}, \int_{R} d y d z \rightarrow \int_{-y_{1}}^{y_{1}} \int_{z_{0}}^{z_{1}}$ $d y d z$, into Eq. (29) we obtain

$$
\begin{aligned}
c(x, y, z)= & Q \sum_{i=0}^{\infty} \sum_{j=0}^{\infty} \frac{Y_{i}(y) Z_{i}(y)}{N_{y}\left(\gamma_{i}\right) N_{z}\left(\eta_{j}\right)} Y_{i}\left(y_{s}\right) Z_{j}\left(z_{s}\right) \\
& \times \exp \left[-\gamma_{i}^{2} s_{y}(x)-\eta_{j}^{2} s_{z}(x)\right]
\end{aligned}
$$

Where: $s_{y}(x)=\int_{0}^{x} a\left(x^{\prime}\right) d x^{\prime}$ and $s_{z}(x)=\int_{0}^{x} b\left(x^{\prime}\right) d x^{\prime}$.

Alternatively, Eq. (30) can be re-written as the product of two functions:

$c(x, y, z)=c_{y}(x, y) c_{z}(x, z)$ 
$c_{y}(x, y)=\sum_{i=0}^{\infty} \frac{Y_{i}(y)}{N_{y}\left(\gamma_{i}\right)} \int_{-y_{1}}^{y_{1}} Y_{i}(y) c_{y}(0, y) d y \exp \left[-\gamma_{i}^{2} s_{y}(x)\right]$

$c_{y}(0, y)=\delta\left(y-y_{s}\right)$

$c_{z}(x, z)=\sum_{j=0}^{\infty} \frac{Z_{j}(z)}{N_{z}\left(\eta_{j}\right)} \int_{z_{0}}^{z_{1}} u(z) Z_{j}(z) c_{z}(0, z) d z \exp \left[-\eta_{j}^{2} s_{z}(x)\right]$

$c_{z}(0, z)=\frac{Q}{u(z)} \delta\left(z-z_{s}\right)$

The Eq. (30) or Eqs. (31)-(33a,b) gives the formal analytical solution for the atmospheric equation (Eqs. (6a,b)-(9)) in a finite media.

\section{Extension of the formal analytical solutions for semi- infinite and infinite region}

Mikhailov and Ozisik (1984) described a systematized procedure to transform the solution for a finite region $\xi_{0} \leq \xi \leq \xi_{1}$ in a solution for a semi-infinite region $\xi_{0} \leq \xi<\infty$. We summarize the systematized procedure of Mikhailov and Ozisik (1984) as follows:

The general representative formula for a finite domain is given by:

$\theta(\bar{x}, \xi)=\sum_{i=0}^{\infty} \frac{\phi_{i}(z)}{N\left(\mu_{i}\right)} \int_{\xi_{0}}^{\xi_{1}} \bar{u}(\xi) \phi_{i}(\xi) \theta(0, \xi) d \xi \exp \left[-\mu_{i}^{2} s(\bar{x})\right]$

Where: $\phi_{i}(z), \mu_{i}$ and $N\left(\mu_{i}\right)$ respectively are, the eigenfunctions, eigenvalues and norm of the following general Sturm-Liouville equation in a finite domain:

$\frac{d}{d \xi}\left[\bar{K}(\xi) \frac{d \phi}{d \xi}\right]+\left[\mu^{2} \bar{u}(\xi)-\bar{d}(\xi)\right] \phi=0 ; \quad \xi_{0} \leq \xi \leq \xi_{1}$

The discrete eigenvalues $\mu_{i}(i=1,2, \ldots, \infty)$ will be replaced by continuous eigenvalues from zero to infinity when the domain is extended to infinite, i.e, $\xi_{1} \rightarrow \infty$. The relation to transform the solution for a finite region $\xi_{0} \leq \xi \leq \xi_{1}$ in the solution for a semiinfinite region $\xi_{0} \leq \xi<\infty$ can be established using the asymptotic behavior of the solution of the Sturm-Liouville equation, resulting:

$\sum_{i=0}^{\infty} F\left(\mu_{i}, \bar{x}, \xi\right) \rightarrow \frac{1}{\pi} \int_{\mu=0}^{\infty}\left(\int_{\xi_{0}}^{\infty} \sqrt{\bar{u}(\xi) / \bar{K}(\xi)} d \xi\right) F(\mu, \bar{x}, \xi) d \mu$

Using Eq. (36) in Eq. (34) we obtain the general formula for a semi-infinite domain $\left(\xi_{1} \rightarrow \infty\right)$ :

$\theta(\bar{x}, \xi)=\frac{1}{\pi} \int_{\xi^{\prime}=\xi_{0}}^{\infty} \bar{u}\left(\xi^{\prime}\right) \theta\left(0, \xi^{\prime}\right) \Omega\left(x, \xi, \xi^{\prime}\right) d \xi^{\prime}$

$\Omega\left(\bar{x}, \xi, \xi^{\prime}\right)=\int_{\mu=0}^{\infty} G(\mu) \phi(\xi) \phi\left(\xi^{\prime}\right) \exp \left[-\mu^{2} \bar{x}\right] d \mu$

$$
G(\mu)=\lim _{\xi_{1} \rightarrow \infty} \frac{\int_{\xi_{0}}^{\xi_{1}} \sqrt{\bar{u}(\xi) / \bar{K}(\xi)} d \xi}{\int_{\xi_{0}}^{\xi_{1}} \bar{u}(\xi) \phi^{2}(\xi) d \xi}
$$

\subsection{Infinite domain formula for $c_{y}(x, y)$}

The procedure described before (Eqs. (36)-(38)) is applied to Eq. (32a,b). Thus, considering the equivalencies: $\bar{x} \rightarrow x, \xi \rightarrow y, \xi_{0} \rightarrow-y_{1}$, $\xi_{1} \rightarrow y_{1}, \bar{u}(\xi) \rightarrow 1, \bar{K}(\xi) \rightarrow 1, \mu \rightarrow \gamma, \quad \phi \rightarrow Y=\cos \left[\gamma\left(y+y_{1}\right)\right]$, $\theta\left(0, \xi^{\prime}\right) \rightarrow c_{y}\left(0, y^{\prime}\right)=\delta\left(y^{\prime}-y_{s}\right)$, results:

$G(\gamma)=\lim _{y_{1} \rightarrow \infty} \frac{1}{y_{1}} \int_{-y_{1}}^{y_{1}} 1 d \xi=2$

$$
\begin{aligned}
\Omega\left(x, y, y^{\prime}\right)= & \int_{\gamma=0}^{\infty} \cos \left[\gamma\left(y+y_{1}\right)\right] \cos \left[\gamma\left(y^{\prime}+y_{1}\right)\right] \\
& \times \exp \left[-\gamma^{2} s_{y}(x)\right] d \gamma
\end{aligned}
$$

An equivalent integral is found in Gradshteyn and Ryshik (1980):

$$
\begin{aligned}
& \int_{0}^{\infty} \exp \left(-\beta x^{2}\right) \cos (a x) \cos (b x) d x \\
& =\frac{1}{4} \sqrt{\frac{\pi}{\beta}}\left(\exp \left[-\frac{(a-b)^{2}}{4 \beta}\right]+\exp \left[-\frac{(a+b)^{2}}{4 \beta}\right]\right)
\end{aligned}
$$

Then,

$$
\begin{aligned}
\Omega\left(x, y, y^{\prime}\right)= & \frac{1}{4} \sqrt{\frac{\pi}{s_{y}(x)}}\left(\exp \left[-\frac{\left(y-y^{\prime}\right)^{2}}{4 s_{y}(x)}\right]\right. \\
& \left.+\exp \left[-\frac{\left(y+2 y_{1}+y^{\prime}\right)^{2}}{4 s_{y}(x)}\right]\right) \\
c_{y}(x, y)= & \frac{2}{\pi} \int_{y^{\prime}=-y_{1}}^{\infty} \delta\left(y^{\prime}-y_{s}\right) \Omega\left(x, y, y^{\prime}\right) d y^{\prime} \\
= & \frac{1}{2 \sqrt{\pi s_{y}(x)}}\left(\exp \left[-\frac{\left(y-y_{s}\right)^{2}}{4 s_{y}(x)}\right]\right. \\
& \left.+\exp \left[-\frac{\left(y+2 y_{1}+y_{s}\right)^{2}}{4 s_{y}(x)}\right]\right)
\end{aligned}
$$

Therefore, when $-y_{1} \rightarrow-\infty$, we obtain the formula for $c_{y}(x, y)$ in an infinite domain:

$c_{y}(x, y)=\frac{1}{2 \sqrt{\pi s_{y}(x)}} \exp \left[-\frac{\left(y-y_{s}\right)^{2}}{4 s_{y}(x)}\right]$

\subsection{Semi-infinite domain formula for $c_{z}(x, z)$}

Similarly, applying in Eq. (33a,b) the equivalencies: $\bar{x} \rightarrow x, \xi \rightarrow z$, $\xi_{0} \rightarrow z_{0}, \quad \xi_{1} \rightarrow \infty, \bar{u}(\xi) \rightarrow u(z), \bar{K}(\xi) \rightarrow K(z), \mu \rightarrow \eta, \phi \rightarrow Z$, $\theta\left(0, \xi^{\prime}\right) \rightarrow c_{z}\left(\mathbf{0}, z^{\prime}\right)=\left(Q / u\left(z^{\prime}\right)\right) \delta\left(z^{\prime}-z_{s}\right)$ results, 
$\Omega\left(x, z, z^{\prime}\right)=\int_{\eta=0}^{\infty} G(\eta) Z(z) Z\left(z^{\prime}\right) \exp \left[-\eta^{2} s_{z}(x)\right] d \eta$

$c_{z}(x, z)=\frac{Q}{\pi} \int_{z^{\prime}=z_{0}}^{\infty} \delta\left(z^{\prime}-z_{s}\right) \Omega\left(x, z, z^{\prime}\right) d z^{\prime}=\frac{Q}{\pi} \Omega\left(x, z, z_{s}\right)$

Where: $G(\eta)$ must be obtained from Eq. (39).

\section{Analysis}

The analytical expression (Eq. (31)) gives the unified solutions for situations of finite (Eqs. (32a,b) and (33a,b)) and infinite/semiinfinite (Eqs. (45) and (47)) domain of the steady-state atmospheric diffusion equation.

In the items 5.1, 5.2, and 5.3 will be shown that the existing closed-form analytical solutions for finite and infinite/semi-infinite domain available in the literature are special cases of the generalized analytical solutions developed above.

Furthermore, in the case of finite domain (item 5.4) a better solution was obtained when a more realistic and modern description of the velocity and turbulent eddy profiles available in the literature are applied to Copenhagen and Prairie Grass experiments showing the performance and robustness of the present analytical solution.

\subsection{Gaussian plume equation: infinite transversal domain and semi-infinite vertical domain}

In the Gaussian plume model, the transversal domain $y$ is $(-\infty$, $\infty$ ) and semi-infinite for the vertical coordinate $z$. The mean wind velocity is considered constant, $u(z)=U$, and the diffusion coefficients are related to the standard deviation as follows:

$K_{y y}(x, y)=U \frac{1}{2} \frac{d\left[\sigma_{y}^{2}(x)\right]}{d x} ;$

$K_{z z}(x, z)=U \frac{1}{2} \frac{d\left[\sigma_{z}^{2}(x)\right]}{d x}$

Therefore, from Eq. (3a,b):

$a(x)=\frac{1}{2} \frac{d\left[\sigma_{y}^{2}(x)\right]}{d x} ;$

$b(x)=\frac{1}{2} \frac{d\left[\sigma_{z}^{2}(x)\right]}{d x} ; K(z)=U$

Thus,

$s_{y}(x)=\int_{0}^{x} a(x \prime) d x^{\prime}=\frac{1}{2} \sigma_{y}^{2}(x)$

$s_{z}(x)=\int_{0}^{x} b\left(x^{\prime}\right) d x^{\prime}=\frac{1}{2} \sigma_{z}^{2}(x)$

Then, Eq. (45) becomes:

$c_{y}(x, y)=\frac{1}{\sqrt{2 \pi} \sigma_{y}(x)} \exp \left[-\frac{\left(y-y_{s}\right)^{2}}{2 \sigma_{y}^{2}(x)}\right]$
The detailed procedure to obtain $c_{z}(x, z)$ under the Gaussian model assumptions is given in Appendix B of the supplementary information, and results in:

$c_{z}(x, z)=\frac{1}{\sqrt{2 \pi} U \sigma_{z}(x)}\left(\exp \left[-\frac{\left(z-z_{s}\right)^{2}}{2 \sigma_{z}^{2}(x)}\right]+\exp \left[-\frac{\left(z+z_{s}\right)^{2}}{2 \sigma_{z}^{2}(x)}\right]\right)$

The classical formula of the Gaussian plume equation is obtained from Eqs. (51) and (52) when inserted in Eq. (31). It is important to note that Eq. (52) corresponds to the case of total reflection at the surface. The total absorption surface solution can be obtained using the Dirichlet boundary condition in Eq. (7a).

\subsection{Polynomial description for the velocity and the turbulent diffusivity: finite media}

In this case the velocity and the eddy diffusivity profiles are specified as:

$u(z)=u_{r} z^{\alpha}$

$K(z)=K_{r} z^{\beta}$

$K_{z z}(x, z)=b(x) K_{r} z^{\beta}$

Where: $u_{r}$ and $K_{r}$ are constants. Setting $z_{0}=0$, the solution of the eigenvalue problem (Eq. $(16 \mathrm{a}-\mathrm{c})$ ) is easily obtained from the generalized Bessel solution (Ozisik, 1980). Using the boundary condition at $z=0$ and considering that the solution remains finite, results:

$Z_{j}= \begin{cases}1 ; & j=0 \\ z^{(1-\beta) / 2} J_{-\nu}\left[\omega_{j} z^{p / 2}\right] ; & j=1,2,3, \ldots\end{cases}$

$\nu=\frac{1-\beta}{p}$

$\omega_{j}=\frac{\eta_{j}\left(u_{r} / K_{r}\right)^{1 / 2}}{p / 2}$

$p=\alpha-\beta+2$

From the boundary condition at $z=z_{1}$ the transcendental equations are obtained,

$J_{-\nu+1}\left[\omega_{j} z^{p / 2}\right]=0$

And, from Eq. (19), results the norm:

$N\left(\eta_{j}\right)=\left\{\begin{array}{l}\frac{u_{r}}{(\alpha+1)} z_{1}^{\alpha+1} ; j=0 \\ \frac{u_{r}}{p} z_{1}^{p} J_{-\nu}^{2}\left(\omega_{j} z_{1}^{p / 2}\right) ; j=1,2,3, \ldots\end{array}\right.$

Then, Eq. (33a,b) can be invoked resulting in:

$$
\begin{aligned}
c_{z}(x, z)= & \frac{(\alpha+1)}{u_{r} z_{1}^{\alpha+1}}+\frac{\left(z z_{s}\right)^{(1-\beta) / 2} p}{u_{r} z_{1}^{p}} \sum_{j=1}^{\infty} \frac{J_{-\nu}\left(\omega_{j} z^{p / 2}\right) J_{-\nu}\left(\omega_{j} z_{s}^{p / 2}\right)}{J_{-\nu}^{2}\left(\omega_{j} z_{1}^{p / 2}\right)} \\
& \times \exp \left[-\eta_{j}^{2} s_{z}(x)\right]
\end{aligned}
$$

This expression is similar to that obtained by Sharan and Kumar (2009). In the case of $b(x)=1, s_{z}(x)=x$, the formula corresponds to 
the previous solution derived by Yeh (1975), Demuth (1978) and Lin and Hildemann (1996).

\subsection{Polynomial description for the velocity and the turbulent diffusivity: semi-finite media}

If the previous results of part 5.2 are used in Eqs. (39) and (40), the following solution is obtained (Appendix $\mathrm{C}$ of the supplementary information):

$c_{z}(x, z)=\frac{Q}{p K_{r} s_{z}(x)}\left(z z_{s}\right)^{(1-\beta) / 2} \exp \left[-\frac{u_{r}\left(z^{p}+z_{s}^{p}\right)}{K_{r} p^{2} s_{z}(x)}\right] I_{-v}\left[\frac{2 u_{r}\left(z z_{s}\right)^{p / 2}}{K_{r} p^{2} s_{z}(x)}\right]$

It is worthy mention that, to the best knowledge of the authors, this is a new generalized formula valid for any $s_{z}(x)$ functional form. The special case with $b(x)=1, s_{z}(x)=x$, corresponds to the previous results obtained by Vaughan (1961), Berlyand (1975), Yeh (1975), Huang (1979) and Lin and Hildemann (1996).

\subsection{Velocity $u(z)$ and eddy turbulent diffusivity $K_{z}(x, z)=b(x) K(z)$ : finite media}

A key issue in dispersion modeling is turbulence parameterization. In general, the functional forms available in the literature for the mean wind speed and the turbulent diffusion coefficient do not allow an analytical solution of the auxiliary eigenvalue problem (Eq. $(16 a-c))$ in a closed-form in terms of known mathematical functions. However, the formal analytical solution can be obtained by integral transform technique as given in Appendix $A$ of the supplementary information.

\section{Results and discussion}

The present formal analytical solution for finite domain was implemented with Mathematica (Wolfram Research, Inc., 2009). For each experiment we found the eigenfunction $\left(Z_{j}\right)$, eigenvalues $\left(\eta_{j}\right)$ and the norm $\left(N_{z}\left(\eta_{j}\right)\right)$ following the procedure described in Appendix $A$ of the supplementary information.

The proposed model is evaluated against the data of the Copenhagen and Prairie Grass diffusion experiments. The quantitative evaluation of the model performance was done using the analysis of the convergence behavior of the results and scatter diagrams for the observed and predicted ground-level crosswind integrated concentrations. Besides this, the statistical indices proposed by Hanna et al. (1991) were used to analyze the agreement between the observed $(O)$ and predicted $(P)$ values. These indices are: fractional bias $(F B)$, fractional variance $(F S)$, normalized mean square error (NMSE), correlation coefficient $(R)$ and fraction within a factor of two (FAC2) and are defined as:

$$
\begin{aligned}
& F B=\frac{\bar{O}-\bar{P}}{0.5(\bar{O}+\bar{P})} ; \\
& F S=\frac{\sigma_{O}-\sigma_{P}}{0.5\left(\sigma_{O}-\sigma_{P}\right)} \\
& N M S E 1=\frac{(O-P)^{2}}{\overline{O P}} ; \\
& N M S E 2=\frac{(O-P)^{2}}{\overline{O P}} ;
\end{aligned}
$$

$R=\frac{\overline{(O-\bar{O})(P-\bar{P})}}{\sigma_{O} \sigma_{P}}$

$F A C 2=$ Fraction of data for which $0.5 \leq P / 0 \leq 2$

Where: $\sigma_{O}$ and $\sigma_{P}$ are the standard deviations of observed and predicted quantities respectively. The over bar indicates an average and a perfect model would have the idealized values NMSE, FB, FS equal to zero and $R, F A C 2$ equal to unity.

\subsection{Copenhagen experiments}

The Copenhagen experiments (Gryning and Lyck, 1984) were carried out under neutral and unstable atmospheric conditions. The tracer sulphurhexafluoride was released without buoyancy from a tower at a height of $115 \mathrm{~m}$ (elevated source) and then collected at ground-level positions in up to three crosswind series of tracer sampling units, positioned $2-6 \mathrm{~km}$ from the point release. The site was mainly residential having a roughness length of $0.6 \mathrm{~m}$ (urban area). Table 1 gives the meteorological input data for the complete set of experiments.

Table 2 shows the convergence process for $c_{z}\left(x, z=z_{0}\right) / Q$ in the nine experiments and the comparison of the obtained values with the observed data. The parameterizations for the wind speed and momentum eddy diffusivities developed by Ulke (2000) were used. The eddy diffusivity parameterization retains the effect of shear stress in the atmospheric boundary layer (ABL) including friction velocity as a scaling velocity. The Ulke's parameterizations consider the current understanding of the ABL in a simple and continuous formulation through the different atmospheric conditions, where the stability-dependent function provides a smooth variation between stable and unstable conditions in the ABL. Besides this, includes the effects of mechanical and buoyancy-induced turbulence in a consistent way, in the wind and the eddy diffusivities functional forms.

As explained by Cotta (1993), the analytic nature of the inversion formula allows for a direct testing procedure at each specified position within the medium where the solution is desired. In this way, the convergence of our results is measured using the number of terms in the series solution.

As can be observed in Table 2, some runs required only $N=5$ terms for the convergence at the different positions from $1900 \mathrm{~m}$ to $6000 \mathrm{~m}$. It can be noted that the associated eigenvalue problem proposed in this work greatly improves the convergence behavior, because only $N=10$ terms in the series of the eigenfunctionexpansion allow to reach the convergence with five decimal digits. Besides this, we can note that the convergence rate increases with distance from the source.

Recently, Kumar and Sharan (2010) developed an important analytical solution for dispersion of pollutants in the atmospheric boundary layer, where the eigenfunction-expansion is based on the simple Sturm-Liouville equation and the transformed ordinary

Table 1

Meteorological data for the Copenhagen experiment (Gryning and Lyck, 1984).

\begin{tabular}{llrr}
\hline Run & $u_{* 0}\left(\mathrm{~ms}^{-1}\right)$ & $L(\mathrm{~m})$ & $z_{1}(\mathrm{~m})$ \\
\hline 1 & 0.37 & -46 & 1980 \\
2 & 0.74 & -384 & 1920 \\
3 & 0.39 & -108 & 1120 \\
4 & 0.39 & -173 & 390 \\
5 & 0.46 & -577 & 820 \\
6 & 1.07 & -569 & 1300 \\
7 & 0.65 & -136 & 1850 \\
8 & 0.70 & -72 & 810 \\
9 & 0.77 & -382 & 2090 \\
\hline
\end{tabular}


Table 2

Convergence of the modeled crosswind integrated concentration $c_{z}(x, z) / Q$ and comparison with observed results $\left(10^{-4} \mathrm{sm}^{-2}\right)$ for the Copenhagen experiment, $K(z)=K_{M}(z)$.

\begin{tabular}{lllllll}
\hline Exp & $x(\mathrm{~m})$ & $N=5$ & $N=10$ & $N=20$ & $N=30$ & Obs. \\
\hline 1 & 1900 & 8.01948 & 8.01237 & 8.01237 & 8.01237 & 6.48 \\
& 3700 & 4.86333 & 4.86333 & 4.86333 & 4.86333 & 2.31 \\
2 & 2100 & 4.38475 & 4.27043 & 4.27045 & 4.27045 & 5.38 \\
& 4200 & 3.00266 & 3.00088 & 3.00088 & 3.00088 & 2.95 \\
3 & 1900 & 8.45145 & 8.44943 & 8.44943 & 8.44943 & 8.2 \\
& 3700 & 5.71738 & 5.71738 & 5.71738 & 5.71738 & 6.22 \\
& 5400 & 4.37327 & 4.37327 & 4.37327 & 4.37327 & 4.3 \\
4 & 4000 & 8.11192 & 8.11192 & 8.11192 & 8.11192 & 11.66 \\
5 & 2100 & 7.27894 & 7.27484 & 7.27484 & 7.27484 & 6.72 \\
& 4200 & 5.58711 & 5.58711 & 5.58711 & 5.58711 & 5.84 \\
& 6100 & 4.48995 & 4.48995 & 4.48995 & 4.48995 & 4.97 \\
6 & 2000 & 3.09881 & 3.05236 & 3.05236 & 3.05236 & 3.96 \\
& 4200 & 2.2451 & 2.24486 & 2.24486 & 2.24486 & 2.22 \\
& 5900 & 1.79819 & 1.79819 & 1.79819 & 1.79819 & 1.83 \\
7 & 2000 & 3.60214 & 3.61284 & 3.61284 & 3.61284 & 6.7 \\
& 4100 & 2.47724 & 2.47727 & 2.47727 & 2.47727 & 3.25 \\
& 5300 & 2.10326 & 2.10326 & 2.10326 & 2.10326 & 2.23 \\
8 & 1900 & 4.83142 & 4.83142 & 4.83142 & 4.83142 & 4.16 \\
& 3600 & 3.35113 & 3.35113 & 3.35113 & 3.35113 & 2.02 \\
& 5300 & 2.64399 & 2.64399 & 2.64399 & 2.64399 & 1.52 \\
9 & 2100 & 4.2215 & 4.08918 & 4.0892 & 4.0892 & 4.58 \\
& 4200 & 2.86522 & 2.86262 & 2.86262 & 2.86262 & 3.11 \\
& 6000 & 2.20449 & 2.20439 & 2.20439 & 2.20439 & 2.59 \\
\hline \multirow{4}{*}{9} & & & & & & \\
& & & & & &
\end{tabular}

differential equation is solved by a strategy of eigensystem. Kumar and Sharan (2010) found that the concentration becomes almost constant as the number of terms $(N)$ increases beyond $N=100$ and revealed that $N=250$ is sufficient to obtain the converged results. Wortmann et al. (2005) show a table as illustration of the convergence for the run nine of the Copenhagen experiment (Table 1 from Wortmann et al., 2005), where it is evidenced that there are necessary $N=150$ summed terms in the series to reach a convergence of three decimal figures. However, the authors were not emphatic in the numbers of terms $(N)$ required to reach the convergence for all their results.

The convergence of our series is better than that of Wortmann et al. (2005) and Kumar and Sharan (2010) because is associated directly to the atmospheric diffusion equation. The eigenvalue problem of them do not include information about the diffusion $-K(z)$ and the mean wind velocity $-u(z)$. Both coefficients were considered in our eigenvalue problem (Eq. $(16 a-c)$ ) resulting in a faster convergence rates of our results.

The scatter diagram of the observed and predicted normalized ground-level crosswind integrated concentrations $\left(c_{z}(x, z) / Q\right)$ is shown in Fig. 1 . The momentum eddy diffusivity and wind speed were obtained from Ulke (2000). The comparison shows that the predicted concentrations from the proposed model are in good agreement with the experimental dataset, with a slight underestimation of the observed values.

Table 3 shows the comparison between statistical indices by CITT and those obtained using different procedures for the solution of the advection-diffusion equation, but with the same formulation for the transport and turbulent diffusion processes: case $1 \mathrm{a}, \mathrm{b}-$ comparison with Ulke (2000), case2a, b - comparison with Vilhena et al. (1998) and Kumar and Sharan (2010), case3 - comparison with Wortmann et al. (2005). In order to show the effectiveness of the solutions, the standard statistical performance measures are compared against results available in the literature.

The ADMM model of Vilhena et al. (1998) was obtained considering the wind speed profile parameterized following the OML model (Berkowicz et al., 1986) and the vertical eddy diffusivity by Degrazia et al. (1997). The equations were solved applying Laplace transform technique with numerical inversion, considering

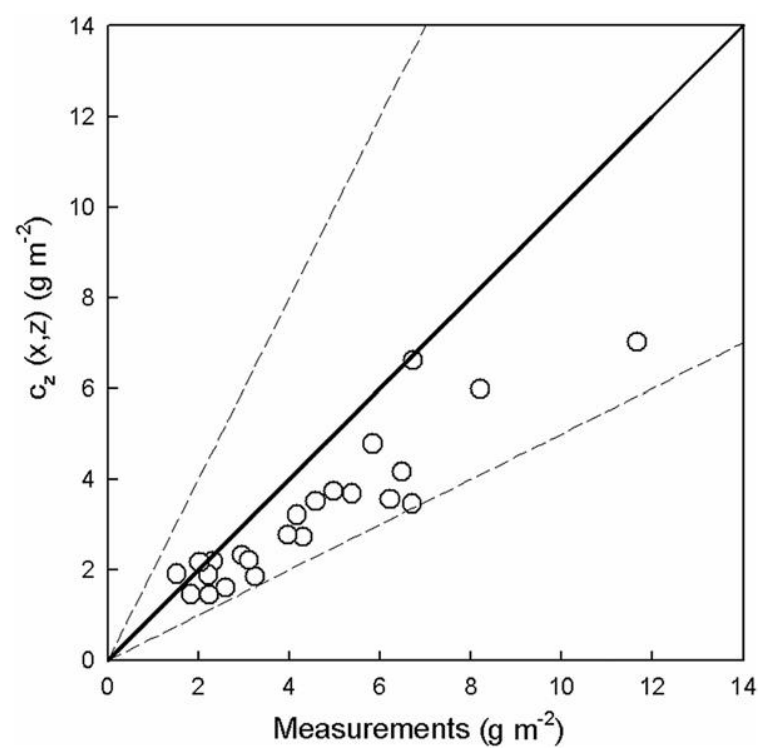

Fig. 1. Observed and predicted crosswind integrated concentration $c_{z}(x, z) / Q$ for Copenhagen experiment, $K(z)=K_{M}(z)$. Middle line in plot is one to one line and outer dashed lines are the lines with a factor of two.

the $\mathrm{ABL}$ as a multi-layer system where the eddy diffusivity and wind are constants in each layer.

Ulke (2000) developed a modeling approach for the dispersion of pollutants released in the atmospheric boundary layer. The model includes a continuous formulation for the transport and turbulent diffusion processes that adequately represents the mechanisms in the various regimes of the atmospheric boundary layer by using a heat eddy diffusivity (MH model) and momentum eddy diffusivity (MM model). The wind speed profiles proposed by Ulke (2000) present an advantageous continuous description for the whole $\mathrm{ABL}$, from stable to unstable stability conditions.

The GILTT technique was proposed by Wortmann et al. (2005) by using Laplace transform technique with analytical inversion. The wind speed profile used has been parameterized following the OML model (Berkowicz et al., 1986) and the vertical eddy diffusivity by Degrazia et al. (1997), like in ADMM model.

An analytical scheme was developed by Kumar and Sharan (2010) to solve the two-dimensional steady-state advection-diffusion considering a horizontal wind speed as a generalized function of

Table 3

Comparison of the statistical indices for crosswind integrated concentration $\left(\mathrm{s} \mathrm{m}^{-2}\right)$ for the simulations of the Copenhagen experiment.

\begin{tabular}{|c|c|c|c|c|c|c|c|}
\hline Case & Model & NMSE1 & NMSE2 & $R$ & $F B$ & FS & FAC2 \\
\hline \multirow[t]{2}{*}{$1 a$} & CITT - MM $^{\text {Present work }}$ & 0.06 & 0.07 & 0.83 & 0.02 & 0.20 & 0.96 \\
\hline & $\mathrm{MM}$ model $^{\mathrm{a}}$ & 0.07 & - & 0.87 & 0.02 & 0.21 & 0.96 \\
\hline \multirow[t]{2}{*}{$1 \mathrm{~b}$} & $\mathrm{CITT}-\mathrm{MH}^{\text {Present work }}$ & 0.16 & 0.20 & 0.88 & 0.33 & 0.41 & 1.00 \\
\hline & MH model $^{\mathrm{b}}$ & 0.20 & - & 0.92 & 0.33 & 0.41 & 1.00 \\
\hline \multirow[t]{2}{*}{$2 a$} & CITT $-\mathrm{KS} 1^{\text {Present work }}$ & 0.08 & 0.09 & 0.86 & 0.13 & 0.35 & 1.00 \\
\hline & $\mathrm{KS} 1^{\mathrm{c}}$ & 0.086 & - & 0.90 & 0.12 & 0.65 & 1.00 \\
\hline \multirow[t]{3}{*}{$2 b$} & CITT - KS2 ${ }^{\text {Present work }}$ & 0.05 & 0.06 & 0.87 & 0.06 & 0.23 & 1.00 \\
\hline & $\mathrm{KS} 2^{\mathrm{d}}$ & 0.075 & - & 0.88 & 0.039 & 0.53 & 1.00 \\
\hline & $\mathrm{ADMM}^{\mathrm{e}}$ & 0.07 & - & 0.90 & 0.06 & 0.23 & 1.00 \\
\hline \multirow[t]{2}{*}{3} & $\mathrm{CITT}^{\mathrm{f}, \text { Present work }}$ & 0.06 & 0.07 & 0.88 & -0.17 & -0.03 & 0.96 \\
\hline & GILTT $^{g}$ & 0.06 & - & 0.92 & -0.14 & -0.02 & 1.00 \\
\hline
\end{tabular}

${ }^{a}$ MM - Momentum eddy diffusivity by Ulke (2000).

b $\mathrm{MH}$ - Heat eddy diffusivity by Ulke (2000).

c KS1 - Kumar and Sharan (2010) with eddy diffusivity by Degrazia et al. (1997).

d KS2 - Kumar and Sharan (2010) with eddy diffusivity by Wortmann et al. (2005).

e ADMM - Vilhena et al. (1998).

${ }^{\text {f }}$ CITT - Eddy diffusivity by Degrazia et al. (1997) with constant velocity.

g GILTT - Wortmann et al. (2005). 
vertical height above the ground as proposed by Gryning et al. (2007). Kumar and Sharan (2010) evaluated their results against Copenhagen diffusion experiment dataset in convective conditions considering different eddy diffusivities. The first one (KS1) is a function only of vertical height as derived by Degrazia et al. (1997). Kumar and Sharan (2010) took the same parameterizations of the wind speed and eddy diffusivity as used by Wortmann et al. (2005) in order to compare their results with the GILTT model (here called KS2).

The analysis of the values in Table 3 indicates that the results from the proposed model by CITT are comparable with those obtained using different procedures previously published in the literature for case1a, b and 2a, b. However, it can be noted a significant discrepancy among GILTT statistical index and results by CITT - KS2. This is more evidenced in the case of FB and FS statistical index, where the GILTT model leads to negative values of $F B$ and $F S$, i.e. a general overestimation of the concentration data is found, while the FB index by CITT - KS2 indicates a slight underestimation of the concentration data. The CITT simulations and GILTT results were similar when it was considered a constant velocity profile and eddy diffusivity by Degrazia et al. (1997) at CITT model, as seen in case 3 in Table 3.

The evaluation of the proposed dispersion model against data from Copenhagen diffusion experiments shows a satisfactory physical behavior and a good overall statistical performance demonstrating the power and accuracy of the formal unified analytical solution.

\subsection{Prairie Grass experiment}

The Prairie Grass diffusion experiments were carried out in a homogeneous field, covered with short grass, in O'Neill, Nebraska, USA, on July and August, 1956 (Barad (ed), 1958). During the experiments, $\mathrm{SO}_{2}$ was released continuously (10 min) from a nearsurface source $(0.5 \mathrm{~m})$ under different meteorological conditions, in 70 experimental runs. The sampling of the released material was done at five concentric arcs at $1.5 \mathrm{~m}$ height and the following distances from the source: 50, 100, 200, 400 and $800 \mathrm{~m}$. In addition, at the $100 \mathrm{~m}$ arc, sampling was made in six towers at nine levels $(0.5,1,1.5,2.5,4.5,7.5,10.5,13.5$ and $17.5 \mathrm{~m})$. Synoptic conditions during each release were also documented. Measurements of temperature and wind speed were carried out in a micrometeorological tower, at $0.25,0.5,1,2,4,8$ and $16 \mathrm{~m}$ height. Upper air observations of meteorological variables with radiosondes and aircraft were also made.

Using the tower measurements, the Richardson number at $2 \mathrm{~m}$ was obtained for each experimental run. The Obukhov length $(L)$ was obtained from the relationship (Businger et al., 1971):

$R i=\frac{\phi_{h}(z / L)}{\phi_{m}^{2}(z / L)} \frac{z}{L}$

Where the non-dimensional gradients for momentum and temperature are those by Wieringa (1980):

$\phi_{m}(z / L)=\left\{\begin{array}{cc}1+6.9 \frac{z}{L} & \frac{z}{L} \geq 0 \\ \left(1-22 \frac{z}{L}\right)^{-1 / 4} & \frac{z}{L}<0\end{array} ;\right.$

$\phi_{h}(z / L)=\left\{\begin{array}{cc}1+9.2 \frac{z}{L} & \frac{z}{L} \geq 0 \\ \left(1-13 \frac{z}{L}\right)^{-1 / 2} & \frac{z}{L}<0\end{array}\right.$

The experimental runs in which the inverse of the Obukhov length was less or equal than $0.01 \mathrm{~m}^{-1}$ were considered as representative of neutral conditions and were used to estimate the roughness length $\left(z_{0}\right)$ of the experimental field. The intercept of a mean squares fit of the wind speed measurements to the logarithmic wind profile for neutral conditions was found and a mean value of $z_{0}=0.008 \mathrm{~m}$ was obtained. The surface friction velocity for each experimental run was estimated by a least square fit of the wind measurements to the surface layer profiles in diabatic conditions using the expression:

$u(z)=u_{* 0}\left\{\frac{1}{k}\left[\ln \frac{z}{z_{0}}-\psi_{m}\left(\frac{z}{L}\right)\right]\right\}$

With

$\psi_{m}\left(\frac{z}{L}\right)= \begin{cases}-6.9 \frac{z}{L} & \frac{z}{L} \geq 0 \\ 2 \ln \left[\frac{(\mu+1)}{2}\right]+\ln \left[\frac{\left(\mu^{2}+1\right)}{2}\right]-2 \arctan \mu+\frac{\pi}{2} \quad \frac{z}{L} \leq 0\end{cases}$

Where $\mu=\phi_{m}^{-1}(z / L)$ and $k=0.41$ is the von Karman's constant.

The atmospheric boundary layer height was obtained from upper air data from soundings and airplane measurements. For most of the experimental runs, the height of the bottom of the capping inversion was found from the upper air data using the relationship between depth and pressure change between different levels. The influence of the humidity in the mean temperature of the layer was included. During convective conditions, the boundary layer depth was found from the potential temperature and relative humidity measurements made with aircraft as the height where the lines corresponding to constant potential temperature or relative humidity intercepts an upper layer with a local non-null gradient of the properties. The average of these two heights was then considered as the boundary layer depth. Table 4 summarizes the micrometeorological parameters data obtained with the procedure described above, for unstable Prairie Grass experiments, used in the present work.

The convergence behavior of the longitudinal concentration obtained with the CITT approach considering the wind speed and

Table 4

Meteorological data for the Prairie Grass experiment.

\begin{tabular}{rrlrlllr}
\hline$N^{\circ}$ & Run & $u_{*_{0}}\left(\mathrm{~m} \mathrm{~s}^{-1}\right)$ & $z_{1}(\mathrm{~m})$ & $L(\mathrm{~m})$ & $1 / L\left(\mathrm{~m}^{-1}\right)$ & $z_{1} / L$ & $\mathrm{Q}(\mathrm{g} / \mathrm{s})$ \\
\hline 1 & 1 & 0.19 & 370 & -11.7647 & -0.085 & -31.3 & 81.5 \\
2 & 5 & 0.41 & 775 & -35.7143 & -0.028 & -21.9 & 77.8 \\
3 & 6 & 0.46 & 880 & -83.3333 & -0.012 & -10.4 & 89.5 \\
4 & 7 & 0.33 & 630 & -7.14286 & -0.14 & -85.6 & 89.9 \\
5 & 8 & 0.32 & 610 & -23.8095 & -0.042 & -25.7 & 91.1 \\
6 & 9 & 0.49 & 925 & -30.303 & -0.033 & -30.8 & 92.0 \\
7 & 10 & 0.34 & 655 & -9.09091 & -0.11 & -72.8 & 92.1 \\
8 & 11 & 0.52 & 995 & -71.4286 & -0.014 & -14.5 & 95.9 \\
9 & 12 & 0.56 & 1060 & -43.4783 & -0.023 & -24.7 & 99.1 \\
10 & 15 & 0.25 & 900 & -6.66667 & -0.15 & -138.0 & 95.5 \\
11 & 16 & 0.26 & 940 & -3.33333 & -0.3 & -283 & 93.0 \\
12 & 19 & 0.45 & 580 & -14.2857 & -0.07 & -41.0 & 101.8 \\
13 & 20 & 0.64 & 790 & -47.619 & -0.021 & -17.0 & 101.2 \\
14 & 25 & 0.21 & 600 & -5.88235 & -0.17 & -101.0 & 101.4 \\
15 & 26 & 0.45 & 860 & -33.3333 & -0.03 & -26.0 & 97.6 \\
16 & 27 & 0.43 & 1185 & -40.0000 & -0.025 & -30.0 & 98.8 \\
17 & 30 & 0.49 & 1350 & -40.0000 & -0.025 & -34.0 & 98.4 \\
18 & 34 & 0.64 & 690 & -58.8235 & -0.017 & -12.0 & 97.4 \\
19 & 43 & 0.38 & 1000 & -12.1951 & -0.082 & -82.0 & 98.9 \\
20 & 44 & 0.43 & 1470 & -29.4118 & -0.034 & -50.0 & 100.7 \\
21 & 45 & 0.41 & 1130 & -90.9091 & -0.011 & -13.0 & 100.8 \\
22 & 47 & 0.25 & 480 & -5.88235 & -0.17 & -81.5 & 103.1 \\
23 & 49 & 0.47 & 480 & -29.4118 & -0.034 & -16.0 & 102.0 \\
24 & 50 & 0.47 & 710 & -35.7143 & -0.028 & -20.0 & 102.8 \\
25 & 51 & 0.48 & 910 & -38.4615 & -0.026 & -24.1 & 102.4 \\
26 & 57 & 0.49 & 1050 & -208.333 & -0.0048 & -5.0 & 101.5 \\
27 & 62 & 0.36 & 680 & -41.6667 & -0.024 & -16.1 & 102.1 \\
\hline & & & & & & &
\end{tabular}


Table 5

Convergence of the modeled crosswind integrated concentration $c_{z}(x, z)$ and comparison with observed results $\left(\mathrm{g} \mathrm{m}^{-2}\right)$ for the Prairie Grass experiment.

\begin{tabular}{lrllllll}
\hline Exp. & $x(\mathrm{~m})$ & $N=10$ & $N=20$ & $N=30$ & $N=40$ & $N=50$ & Obs. \\
\hline 8 & 50 & 3.96621 & 5.26775 & 5.27368 & 5.27311 & 5.27311 & 5.4194 \\
& 100 & 2.89076 & 3.26775 & 3.2681 & 3.2681 & 3.2681 & 2.8139 \\
& 200 & 1.74277 & 1.785 & 1.785 & 1.785 & 1.785 & 1.2686 \\
& 400 & 0.889993 & 0.890812 & 0.890812 & 0.890812 & 0.890812 & 0.4364 \\
& 800 & 0.41981 & 0.41981 & 0.41981 & 0.41981 & 0.41981 & 0.1568 \\
12 & 50 & 1.75213 & 3.15366 & 3.33378 & 3.33845 & 3.33839 & 3.5384 \\
& 100 & 1.44601 & 2.09237 & 2.11722 & 2.11732 & 2.11732 & 2.0439 \\
& 200 & 1.02597 & 1.19171 & 1.19233 & 1.19233 & 1.19233 & 1.1626 \\
& 400 & 0.595719 & 0.611778 & 0.611779 & 0.611779 & 0.611779 & 0.5276 \\
& 800 & 0.293678 & 0.293933 & 0.293933 & 0.293933 & 0.293933 & 0.224 \\
30 & 50 & 1.69618 & 3.27543 & 3.5619 & 3.57982 & 3.5803 & 4.4259 \\
& 100 & 1.43573 & 2.23989 & 2.29092 & 2.29153 & 2.29153 & 2.4331 \\
& 200 & 1.05957 & 1.30201 & 1.3041 & 1.3041 & 1.3041 & 1.1622 \\
& 400 & 0.642566 & 0.673733 & 0.673739 & 0.673739 & 0.673739 & 0.4184 \\
& 800 & 0.323514 & 0.324377 & 0.324377 & 0.324377 & 0.324377 & 0.2352 \\
44 & 50 & 1.91486 & 3.65744 & 3.958 & 3.97657 & 3.9771 & 4.9327 \\
& 100 & 1.61152 & 2.48324 & 2.53399 & 2.53455 & 2.53455 & 2.5191 \\
& 200 & 1.17789 & 1.43317 & 1.43506 & 1.43506 & 1.43506 & 1.248 \\
& 400 & 0.705075 & 0.736278 & 0.736282 & 0.736282 & 0.736282 & 0.5 \\
& 800 & 0.350815 & 0.351597 & 0.351597 & 0.351597 & 0.351597 & 0.1552 \\
\hline
\end{tabular}

eddy diffusivity profiles proposed by Ulke (2000) is shown in Table 5. Convergence to the sixth significant digits was achieved for truncation orders as low as $N=30$, for the position $x \geq 100 \mathrm{~m}$ and at experiments where the boundary layer heights were bellow $1000 \mathrm{~m}$. For positions closer to the source $(x<100 \mathrm{~m})$ and boundary layer heights bellow $1000 \mathrm{~m}$, six significant figures are achieved at higher orders $(N=50)$, while for experiments where the boundary layer heights were above $1000 \mathrm{~m}$ only four significant digits are obtained with this truncation orders.

The performance of the model is shown in Table 6 and in Fig. 2. Analyzing the statistical indices in Table 6 it is possible to notice that the proposed CITT model can adequately simulate dispersion in convective conditions for near-surface sources. The NMSE, FB and $F S$ values are close to zero and $R$ and FAC2 are relatively near to 1 . A more detailed inspection of the Table 6 allows concluding that the proposed model presents better values of the statistical indices than the presented by Buske et al. (2007) that used different types of parameterizations for the eddy diffusivities and a polynomial description of the mean speed velocity.

Fig. 2 shows the scatter diagram of the observed and predicted crosswind integrated concentrations for the proposed CITT model using the momentum eddy diffusivity and the velocity profile parameterizations developed by Ulke (2000). The analysis of the results shows a reasonably good agreement between the values computed by the models and the experimental ones. However, it can be observed that the model results tend to underestimate the experimental data near the source, around $x$ equal $50 \mathrm{~m}$, and to overestimate the data for positions far from the source $(x>100 \mathrm{~m})$. It can be due to the eddy diffusivity parameterization behavior, that did not consider the variation as a generalized function of the downwind distance $x$ and the height $z$. This situation was not explored in the work, because we understand that this subject will be treated in a future paper.

\section{Table 6}

Comparison of the statistical indices for crosswind integrated concentration $\left(\mathrm{g} \mathrm{m}^{-2}\right)$ obtained for the simulation of the Prairie Grass experiment.

\begin{tabular}{llllrrr}
\hline Eddy diffusivity model & NMSE1 & NMSE2 & \multicolumn{1}{l}{ R } & \multicolumn{1}{c}{ FB } & \multicolumn{1}{c}{ FS } & FAC2 \\
\hline GITT - MM & 0.04 & 0.08 & 0.96 & -0.09 & 0.13 & 0.79 \\
Degrazia et al. (1997), $^{\mathrm{a}}$ & 0.64 & - & 0.83 & 0.31 & 0.46 & 0.68 \\
Similarity theory $^{\mathrm{a}}$ & 0.74 & - & 0.75 & 0.09 & -0.14 & 0.80 \\
Troen-Mahrt $^{\mathrm{a}}$ & 1.20 & - & 0.82 & 0.55 & 0.69 & 0.67 \\
\hline
\end{tabular}

${ }^{\mathrm{a}}$ Buske et al. (2007).

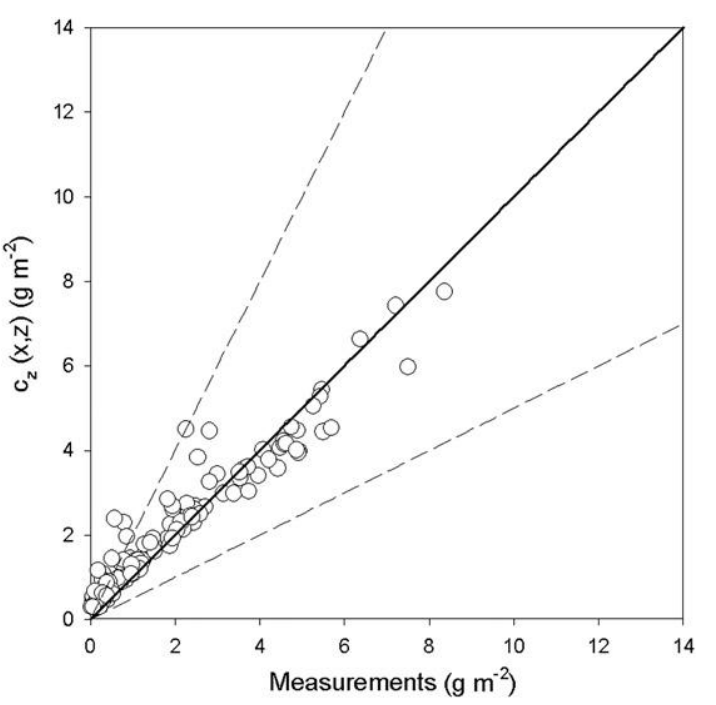

Fig. 2. Observed and predicted crosswind integrated concentration $c_{z}(x, z) / Q$ for the Prairie Grass experiment, $K(z)=K_{M}(z)$. Middle line in plot is one to one line and outer dashed lines are the lines with a factor of two.

\section{Conclusions}

The three-dimensional formulation of the steady-state atmospheric diffusion equation was solved analytically using the classic integral transform technique. The solution was established for an arbitrary mean wind speed depending on the vertical coordinate $(z)$ and a generalized separable functional form for the eddy diffusivities in terms of the longitudinal $(x)$ and vertical coordinates $(z)$.

The formal analytical solution for a finite domain was extended for a semi-infinite and infinite domain exploring a unified procedure from the associated eigenvalue problem to the original advective-diffusive atmospheric equation.

The treated examples showed that previous closed-form analytical solutions available in the literature for both finite and semi-infinite/infinite media are part of the unified analytical solution of the present work. It should be noticed that an original closed-form analytical solution was developed for the case of a polynomial description for the velocity and turbulent coefficient on semi-infinite media.

For generalized situations in a finite region, where the mean wind speed and the turbulent diffusion coefficient are described by functional forms existing in the literature, the unified analytical solution showed to be effective when simulating the Copenhagen and Prairie Grass experiments.

It can be noted that the effect of the associated eigenvalue problem in the solution formula greatly improves the convergence behavior in the series of the eigenfunction-expansion. It was showed in the case of simulation of the Copenhagen experiments that were only necessary $N=10$ terms in the series solution expansion to reach a convergence with six significant digits.

The same precision was achieved with $N=30$ terms in the series solution for Prairie Grass experiments for the position $x \geq 100 \mathrm{~m}$. For positions closer to the source $(x<100 \mathrm{~m}) N=50$ terms were required to achieve four or six significant digits in the results, depending on the height of the boundary layer. The proposed model presents better values of the statistical indices than the presented by Buske et al. (2007) that used different types of parameterizations for the eddy diffusivities and a polynomial description of the mean speed velocity.

The proposed method is a valuable tool to simulate dispersion processes in the atmosphere and is useful to evaluate the 
performance of different turbulence parameterizations in an easy way as well as to obtain estimated concentration with little computational cost. It is important to highlight also that an attraction of the present analytic methodology is the automatic global error control in the computational algorithmic and estimation offers the useful feature of working within a user prescribed accuracy. That characteristics associated to the improvement of the convergence solution are essentials for applications in inverses problems, such as treated by Storch et al. (2007) for the estimation of micrometeorological parameters.

\section{Acknowledgments}

The authors acknowledge the support of the Brazilian National Research Council (CNPq), Carlos Chagas Filho Foundation for Research Support of Rio de Janeiro State (FAPERJ) and the partial support of the UBACyT X224 and 20020100101013 projects financed by the University of Buenos Aires and PICT2008-1739 by the ANPCyT, Argentina.

We also like to thanks to Mr. Miguel Pérez Guerrero for his dedication and collaboration in the preparation of the manuscript.

\section{Appendix. Supplementary material}

Supplementary data associated with this article can be found, in the online version, at doi:10.1016/j.atmosenv.2012.03.015.

\section{References}

Almeida, G.L., Pimentel, L.C.G., Cotta, R.M., 2008. Integral transform solutions for atmospheric pollutant dispersion. Environmental Modeling \& Assessment 13, 53-65.

Bailey, P.B., Gordon, M.K., Shampine, L.F., 1978. Automatic solution of the SturmLiouville problem. ACM Transactions on Mathematical Software (TOMS) 4 , 193-208.

Barad, M.L. (Ed.), 1958. Project Prairie Grass, A Field Program in Diffusion, Geophysical Research Papers No. 59, Vol. I \& II. Air Force Cambridge Research Centre, Bedford, Mass.

Berkowicz, R.R., Olesen, H.R., Torp, U., 1986. The Danish Gaussian air pollution mode (OML). Description, test and sensitivity analysis in view of regulatory applications. In: De Wispeleare, C., Schiermeirier, F.A., Gillani, N.V. (Eds.), Air Pollution Modeling and Its Application. Plenum Publishing Corporation, 453-480 pp.

Berlyand, M.Y., 1975. Contemporary Problems of Atmospheric Diffusion and Pollution of the Atmosphere (NERC, Trans.). USEPA, Raleigh, NC, USA, 448 pp.

Bessagnet, B., Menut, L., Curci, G., Hodzic, A., Guillaume, B., Liousse, C., Moukhtar, S. Pun, B., Seigneur, C., Schulz, M., 2008. Regional modeling of carbonaceous aerosols over Europe - focus on secondary organic aerosols. Journal of Atmospheric Chemistry 61, 175-202.

Bosanquet, C.H., Pearson, J.L., 1936. The spread of smoke and gases from chimneys Transactions of the Faraday Society 32, 1249-1263.

Businger, J.A., Wyngaard, J.C., Izumi, Y., Bradley, E.F., 1971. Flux-profile relationships in the atmospheric surface layer. Journal of the Atmospheric Sciences 28, 181-189.

Buske, D., Vilhena, M.T., Moreira, D.M., Tirabassi, T., 2007. Simulation of pollutant dispersion for low wind conditions in stable and convective planetary boundary layer. Atmospheric Environment 41, 5496-5501.

Byun, D.W., Ching, J.K.S., 1999. Science Algorithms of the EPA Models-3 Community Multiscale Air Quality (CMAQ) Modelling System. U.S. EPA/600/R-99/030.

Byun, D.W., Lacser, A., Yamartino, R., Zannetti, P., 2003. Eulerian dispersion models In: Zannetti, P. (Ed.), Chapter 10 of Air Quality Modeling- Theories, Methodologies, Computational Techniques, and Available Databases and Software. Fundamentals, vol. I. Published by The EnviroComp Institute and the Air \& Waste Management Association, pp. 213-292.

Carruthers, D.J., McKeown, A.M., Hall, D.J., Porter, S., 1999. Validation of ADMS against wind tunnel data of dispersion from chemical warehouse fires. Atmospheric Environment 33, 1937-1953.

Cassol, M., Wortmann, S., Rizza, U., 2009. Analytic modeling of two-dimensional transient atmospheric pollutant dispersion by double GITT and Laplace transform techniques. Environmental Modelling \& Software 24, 144-151.

Cotta, R.M., 1993. Integral Transforms in Computational Heat and Fluid Flow. CRC Press, Boca Raton, FL, 340 pp.

Davies, D.R., 1947. Turbulence and diffusion in the lower atmosphere with particular reference to the lateral effect. Proceedings of the Royal Society of London. Series A, Mathematical and Physical Sciences 190, 232-244.

Degrazia, G.A., Campos Velho, H.F., Carvalho, J.C., 1997. Non-local exchange coefficients for the convective boundary layer derived from spectral properties. Contributions to Atmospheric Physics, 57-64.
Demuth, C., 1978. A contribution to the analytical steady solution of the diffusion equation for line sources. Atmospheric Environment 12, 1255-1258.

Duffy, D.G., 2001. Green's Functions with Applications. Chapman \& Hall/CRC.

EPA (Environmental Protection Agency), 2005. Revision to the Guideline on Air Quality Models: Adoption of a Preferred General Purpose (Flat and Complex Terrain) Dispersion Model and Other Revisions. Environmental Protection Agency, North Carolina, U.S, Federal Register/vol. 70, No. 216/Rules and Regulations. Appendix W of 40 CRF Part 51, 45 pp.

Frost, R., 1946. Turbulence and diffusion in the lower atmosphere. Proceedings of the Royal Society of London. Series A 186, 20-35.

Gradshteyn, I.S., Ryshik, I.M., 1980. Tables of Integrals, Series, Products. Academic Press.

Grell, G.A., Peckham, S.E., Schmitz, R., McKeen, S.A., Wilczak, J., Eder, B., 2005. Fully coupled "online" chemistry within the WRF model. Atmospheric Environment 39, 6957-6975.

Gryning, S.E., Lyck, E., 1984. Atmospheric dispersion from elevated sources in an urban area: comparison between tracer experiments and model calculations. Journal of Climate and Applied Meteorology 23, 651-660.

Gryning, S.E., Batchvarova, E., Brümmer, B., Jørgensen, H., Larsen, S., 2007. On the extension of the wind profile over homogeneous terrain beyond the surface boundary layer. Boundary-Layer Meteorology 124, 251-268.

Hanna, S.R., Briggs, G.A., Hosker Jr., R.P., 1982. Handbook on Atmospheric Diffusion. U.S. Dept. of Energy Report COE/TIC-11223, Washington, DC, 107 pp.

Hanna, S.R., Strimaitis, D.G., Chang, J.C., 1991. Hazard Response Modeling Uncertainty (A Quantitative Method). In: In User's Guide for Software for Evaluation of Hazardous Gas Dispersion Models (Sigma Research Corp.. Westford, USA.

Huang, C.H., 1979. A theory of dispersion in turbulent shear flow. Atmospheric Environment 13, 453-463.

Kumar, P., Sharan, M., 2010. An analytical model for dispersion of pollutants from a continuous source in the atmospheric boundary layer. Proceedings of the Royal Society A: Mathematical, Physical and Engineering Sciences 466, 383-406.

Lebedeff, S.A., Hameed, S., 1975. Steady-state solution of the semi-empirical diffusion equation for area sources. Journal of Applied Meteorology 14, 546-549.

Lin, J.S., Hildemann, L.M., 1996. Analytical solutions of the atmospheric diffusion equation with multiple sources and height-dependent wind speed and eddy diffusivities. Atmospheric Environment 30, 239-254.

Llewelyn, R.P., 1983. An analytical model for the transport, dispersion and elimination of air pollutants emitted from a point source. Atmospheric Environment $17,249-256$.

Ma, J., Daggupaty, S.M., 1998. A generalized analytical solution for turbulent dispersion with inhomogeneous wind and diffusion coefficient. Environmental Modeling \& Assessment 3, 239-248.

Marletta, M., Pryce, J.D., 1992. Automatic solution of Sturm-Liouville problems using the Pruess method. Journal of Computational and Applied Mathematics 39, 57-78.

Mikhailov, M.D., Cotta, R.M., 1994. Integral transform solution of eigenvalue problems. Communications Numerical Methods Engineering 10, 827-835.

Mikhailov, M.D., Ozisik, M.N., 1984. Unified Analysis and Solutions of Heat and Mass Diffusion. John Wiley \& Sons, 524 pp.

Mikhailov, M.D., Vulchanov, N.L., 1983. A computational procedure for SturmLiouville problems. Journal of Computational Physics 50, 323-336.

Moreira, D.M., Villena, M.T., Tirabassi, T., Buske, D., Cotta, R., 2005. Near-source atmospheric pollutant dispersion using the new GILTT method. Atmospheric Environment 39, 6289-6294.

Moreira, D.M., Villena, M.T., Buske, D., Tirabassi, T., 2009. The state-of-art of the GILTT method to simulate pollutant dispersion in the atmosphere. Atmospheric Research 92, 1-17.

Naveira-Cotta, C.P., Cotta, R.M., Orlande, H.R.B., Fudym, O., 2009. Eigenfunction expansions for transient diffusion in heterogeneous media. International Journal of Heat and Mass Transfer 52, 5029-5039.

Ozisik, M.N., 1980. Heat Conduction. John Wiley \& Sons, New York, 704 pp.

Park, Y.S., Baik, J.J., 2008. Analytical solution of the advection-diffusion equation for a ground-level finite area source. Atmospheric Environment 42, 9063-9069.

Pasquill, F., Smith, F.B., 1983. Atmospheric Diffusion. John Wiley\& Sons, New York, $437 \mathrm{pp}$.

Pérez Guerrero, J.S., Skaggs, T.H., 2010. Analytical solution for one-dimensional advection-dispersion transport equation with distance-dependent coefficients. Journal of Hydrology 390, 57-65.

Pérez Guerrero, J.S., Pimentel, L.C.G., Skaggs, T.H., Van Genuchten, M.T., 2009a. Analytical solution of advection-diffusion transport equation using change-ofvariable and integral transform. International Journal of Heat and Mass Transfer 52, 3297-3304.

Pérez Guerrero, J.S., Skaggs, T.H., Van Genuchten, M.T., 2009b. Analytical solution for multi-species contaminant transport subject to sequential first-order decay reactions in finite media. Transport in Porous Media 80, 373-387.

Pérez Guerrero, J.S., Skaggs, T.H., Van Genuchten, M.T., 2010. Analytical solution for multi-species contaminant transport in finite media with time-varying boundary conditions. Transport in Porous Media 85, 171-188.

Roberts, O.F.T., 1923. The theoretical scattering of smoke in a turbulent atmosphere. Proceedings of the Royal Society of London. Series A, Containing Papers of a Mathematical and Physical 104, 640-654.

Rounds, W., 1955. Solutions of the two-dimensional diffusion equation. Transactions - American Geophysical Union 36, 395-405. 
Russell, A., Dennis, R., 2000. NARSTO critical review of photochemical models and modeling. Atmospheric Environment 34, 2283-2324.

Seinfeld, J.H., Pandis, S.N., 1998. Atmospheric Chemistry and Physics. John Wiley and Sons, New York, 1326 pp.

Sharan, M., Gupta, S., 2002. Two-dimensional analytical model for estimating crosswind-integrated concentration in a capping inversion: eddy diffusivity as a function of downwind distance from the source. Atmospheric Environment 36, 97-105.

Sharan, M., Kumar, P., 2009. An analytical model for crosswind integrated concentrations released from a continuous source in a finite atmospheric boundary layer. Atmospheric Environment 43, 2268-2277.

Sharan, M., Modani, M., 2005. An analytical study for the dispersion of pollutants in a finite layer under low wind conditions. Pure and Applied Geophysics 162, 1861-1892.

Sharan, M., Modani, M., 2006. A two-dimensional analytical model for the dispersion of air-pollutants in the atmosphere with a capping inversion. Atmospheric Environment 40, 3469-3489.

Sharan, M., Singh, M.P., Yadav, A.K., 1996. A mathematical model for the atmospheric dispersion in low winds with eddy diffusivities as linear functions of downwind distance. Atmospheric Environment 30, 1137-1145.

Smith, F.B., 1957. The diffusion of smoke from a continuous elevated point-source into a turbulent atmosphere. Journal of Fluid Mechanics 2, 49-76.

Storch, R.B., Pimentel, L.C.G., Orlande, H.R.B., Fudym, O., 2007. Identification of micro-meteorological parameters for the characterization of atmospheric boundary layers by inverse problem and integral transform technique. Atmospheric Environment 3, 1-10.

Sutton, O.G., 1932. A theory of eddy diffusion in the atmosphere. Proceedings of the Royal Society of London. Series A 135, 143-165.
Sutton, W.G.L., 1943. On the equation of diffusion in a turbulent medium. Proceedings of the Royal Society of London. Series A, Mathematical and Physical Sciences 182, 48-75.

Sutton, O.G., 1949. The application to micrometeorology of the theory of turbulent flow over rough surfaces. Quarterly Journal of the Royal Meteorological Society $75,335-350$

Sutton, O.G., 1953. Micrometeorology. McGraw-Hill Book Comp., New York, 320 pp Tirabassi, T., Buske, D., Moreira, D.M., Vilhena, M.T., 2008. A two-dimensional solution of the advection-diffusion equation with dry deposition to the ground Journal of Applied Meteorology and Climatology 47, 2096-2104.

Ulke, A.G., 2000. New turbulent parameterization for a dispersion model in the atmospheric boundary layer. Atmospheric Environment 34, 1029-1042.

Vaughan, L.V., 1961. The prediction of atmospheric diffusion by using an eddy diffusivity based on the vertical transfer of heat. Journal of Meteorology 18, 43-49.

Vilhena, M.T., Rizza, U., Degrazia, G.A., Mangia, C., Moreira, D.M., Tirabassi, T., 1998 An analytical air pollution model: development and evaluation. Contributions to Atmospheric Physics 71, 315-320.

Wieringa, J., 1980. A revaluation of the Kansas mast influence on measurements of stress and cup-anemometer over speeding. Boundary-Layer Meteorology 18, 411-430.

Wolfram Research, Inc., 2009. Mathematica. Version 7.0, Champaign, IL.

Wortmann, S., Vilhena, M.T., Moreira, D.M., Buske, D., 2005. A new analytical approach to simulate the pollutant dispersion in the PBL. Atmospheric Environment 39, 2171-2178.

Yeh, G.T., 1975. Green's function of a diffusion equation. Geophysical Research Letters 2, 293-296.

Yih, C.S., 1952. Similarity solution of a specialized diffusion equation. Transactions American Geophysical Union 33, 356-360. 